\title{
VATS thymectomy for early stage thymoma and myasthenia gravis: combined right-sided uniportal and left-sided three-portal approach
}

\author{
Maurizio Infante, Cristiano Benato, Riccardo Giovannetti, Cinzia Bonadiman, Barbara Canneto, \\ Giovanni Falezza, Alessandro Lonardoni, Paola Gandini \\ Department of Thoracic Surgery, University and Hospital Trust of Verona, Verona, Italy \\ Correspondence to: Maurizio Infante, MD. Department of Thoracic Surgery, University and Hospital Trust, Azienda Ospedaliera Universitaria \\ Integrata, P.le A. Stefani, 1, Verona 37126, Italy. Email: maurizio.infante@aovr.veneto.it.
}

\begin{abstract}
The traditional approach to thymectomy requires median sternotomy based on the assumption that it is the best means to achieve adequate resection margins, complete removal of the thymus and clearance of the anterior mediastinal fat. However, in recent years, VATS thymectomy has been gaining acceptance as a means to achieve adequate oncologic results and symptomatic improvement of myasthenic symptoms with less impact on the patient. We have adopted a flexible approach based on the location of the tumor and on whether the patient has myasthenia gravis (MG) or not when planning minimally invasive VATS thymectomy. A preferential approach from the left side is chosen for clinical stage I-II thymomas located on the left side or on the midline in patients without MG, and a bilateral approach (uniportal VATS on the right side and three-portal VATS on the left side) for MG patients with or without thymoma in order to achieve complete clearance of the anterior mediastinal fat on both sides. Such techniques are herewith clearly illustrated in hope that surgeons wishing to endeavor in such an effort will be facilitated.
\end{abstract}

Keywords: VATS thymectomy; uniportal VATS; thymoma; myasthenia gravis (MG)

Received: 23 August 2017; Accepted: 28 August 2017; Published: 18 October 2017.

doi: 10.21037 /jovs.2017.09.01

View this article at: http://dx.doi.org/10.21037/jovs.2017.09.01

\section{Introduction}

Thymoma is a rare tumor accounting for approximately 0.15 new cases per 100,000 persons/year. Its peak incidence is in the $4^{\text {th }}-6^{\text {th }}$ decade of life. Sixty-five percent of the cases are asymptomatic and come to medical attention for other reasons (1-3).

Thymoma is most often a biologically slow-growing tumor with a high probability of cure after surgery. Diseasefree 10 -year survival is $80-90 \%$ for stage I disease and goes down to less than $50 \%$ for stage IV. Overall 10-year survival is $60-90 \%$ according to stage. A spectrum of aggressiveness can be seen from $\mathrm{WHO}$ histological type $\mathrm{A}$ and $\mathrm{AB}$ to type B2-B3 to type C, i.e., thymic carcinoma for which 5-year survival is only $20-25 \%$. Masaoka stage, WHO histological subtype and complete surgical resection influence the clinical outcome independently (3-5).

The surgical approach to the thymus varies considerably across the world. In Europe and North America, the most widely recommended approach rests on transsternal thymectomy, that allows easy access to the anterior mediastinum and good control of major vascular structures (roughly $75 \%$ of the procedures). Alternatively, a lateral thoracotomy is commonly used, while less than $10 \%$ of thymus gland resections are carried out by minimally invasive techniques. In Asia instead approximately 35\% of the procedures are currently carried out by minimally invasive surgery (VATS or robotic) (6).

Arguments in favor of routine trans-sternal thymectomy even for early-stage thymoma are based on the necessity of obtaining wide resection margins, of removing as much ectopic thymus tissue as possible in myasthenia gravis (MG) 
patients, and on the hypothetical risk that a second primary thymoma may develop in the residual thymus gland $(4,7)$. However, an increasing number of reports over the recent years have shown that thymectomy can be accomplished safely and effectively by VATS both for early-stage thymoma and for MG (8-14).

A recently published propensity-matched study on patients undergoing either trans-sternal or minimally invasive thymectomy for stage I-II thymoma showed overall postoperative complications rates to be lower with VATS thymectomy, and suggested that in fact, the majority of complications in the open group were due the complications of median sternotomy (15).

There are no randomized trials that compare minimally invasive and open thymectomy, but VATS thymectomy was compared to the traditional open approach in two recent large meta-analyses $(16,17)$. Both such studies showed significantly reduced postoperative blood loss and blood products requirements, reduced postoperative pain scores, reduced overall complications rates and reduced postoperative hospital stay with VATS thymectomy compared with open thymectomy. Operating-room time was not significantly different between the two techniques.

Most importantly, the probability of achieving microscopically complete resection (i.e., tumor free margins at pathologic examination) and loco-regional recurrence rates were similar with either approach.

Over $50 \%$ of thymoma patients may suffer from a variety of autoimmune diseases, the most common of which are MG, erythroid aplasia and hypogammaglobinemia. Up to $25 \%$ of thymoma patients will have clinically overt MG and an additional $25 \%$ of clinically asymptomatic thymoma patients will have circulating anti-acetylcholinesterase receptor (anti-Ach-R) autoantibodies. Conversely, in 5-15\% of the patients with $M G$, preoperative work up eventually demonstrates an unsuspected thymoma $(18,19)$.

Thymectomy in MG patients may lead to long-term complete long-term remission (35-40\% of patients), or to clinical improvement, i.e., discontinuation of steroids, less need for resorting to immunosuppressive therapy, lower incidence of myasthenic crisis or symptom improvement in another $30-45 \%(12,19)$. Complete removal of the thymus gland and anterior mediastinal fat from neck to diaphragm and phrenic-to-phrenic nerve (so called maximal thymectomy) is required, as microscopic islets of functional thymic tissue can be found throughout the anterior mediastinal fat in over $20 \%$ of the patients. The benefits of maximal thymectomy for MG patients have been recently confirmed in a randomized trial (20).

Numerous technical variations to VATS thymectomy have been described, including bilateral or single-sided approaches from either the left or right hemi-thorax, subxiphoid and trans-cervical approaches (21-23). The choice will vary according to individual surgeon's expertise and preferences.

In this paper, the technical aspects of minimally invasive maximal thymectomy as performed in our department are clearly illustrated in hope that other surgeons wishing to endeavor in such an effort will be facilitated.

\section{Patient selection and workup}

Contrast-enhanced CT scan and FDG-PET are routinely used to evaluate anterior mediastinal tumors in our department. Radiological studies have shown a good correlation between CT scan results and intraoperative findings, and particularly for stage I-II disease there is a high accuracy in determining whether the tumor will be resectable without the need for major vascular resection (24). More advanced cases are often evaluated by MRI.

All patients with suspected thymomas should be evaluated in a multidisciplinary setting with a neurologist in order to exclude MG, and the anesthesiologist should be involved in the preoperative planning. Patents who are not affected by MG do not pose particular management problems and do not need any special preparation.

However, MG patients should be adequately prepared by appropriate pharmacological intervention to improve symptom control as much as possible prior to the operation, in order to reduce the risk of postoperative myasthenic crisis.

\section{Table of equipment preference card}

(I) $\mathrm{CO}_{2}$ insufflation equipment;

(II) A 30-degree, 5-mm scope (allows changing the camera port if necessary without enlarging the incisions and trocars);

(III) Two 5-mm laparoscopic trocars;

(IV) One 10-mm laparoscopic trocar;

(V) Laparoscopic graspers;

(VI) A laparoscopic energy device for tissue grasping and sealing;

(VII) Laparoscopic suction-irrigation cannula;

(VIII) Laparoscopic specimen bag. 


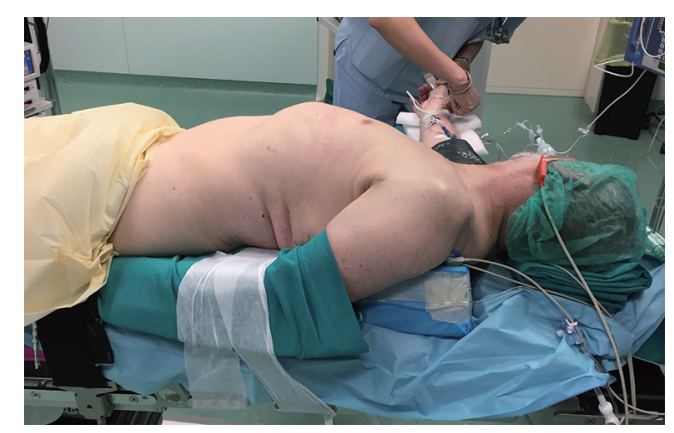

Figure 1 Patient positioning for left-sided VATS approach.

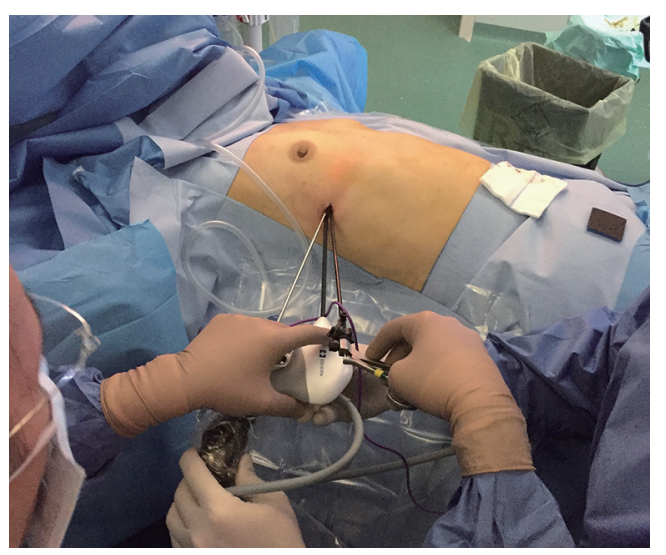

Figure 2 Right-sided uniportal phase in a myasthenia gravis patient.

\section{Patient positioning}

\section{For non-MG thymoma patients}

our preferred approach is through the left hemi-thorax, provided that the tumor is within reach (i.e., on the leftside or midline), as there is better control of the innominate vein along its entire course, and easier access to the cervical horns without interference from the right mammary vein.

The patient is placed in a semi-supine position, closer to the left border of the table, with the left hemithorax lifted by a soft gel shoulder roll. The left arm is gently flexed posteriorly and rests on the table plane in order to fully expose the hemithorax (Figure 1).

Tumors that are frankly located in the right hemithorax are approached from the right side and the positioning is specular.

\section{For MG patients}

The patient will again lie closer to the left border of the table as most dissection maneuvers will be carried out through the left side. However, in this case the entire chest of the patient will be lifted from the table plane by placing 2 gel rolls or similar aide longitudinally underneath the dorsum, the right arm being simply widely abducted to allow the anesthesiologist to access the venous lines and pressure cuff as needed. Such positioning allows the surgeon to perform the first step of the dissection on the right side without the need for subsequent patient re-positioning and re-draping (Figure 2). The patent's hips must be secured to allow tilting of the table as necessary.

\section{Nurse and assistant positioning}

The scrub nurse stands on opposite side with respect to the operating surgeon, i.e., on the right side for all the duration of the operation if the thymectomy is carried out through the left hemi-thorax, or vice versa. The assistant will be standing beside the operating surgeon all the time.

\section{Procedure}

It is by no means possible to reach the opposite cardiophrenic angle and remove all of the anterior mediastinal fat as with the trans-sternal thymectomy by a one-sided VATS approach. Therefore, a bilateral VATS approach is routinely planned for MG patients in our department.

\section{First step: right side}

A single 2-cm incision is made in the $5^{\text {th }}$ intercostal space on the mid-axillary line (Figure 2). The cardiophrenic angle is viewed directly and the mediastinal fat anterior to the right phrenic nerve at that level can be grasped and dissected free from the phrenic nerve (Figure 3).

The dissection is pushed along the anterior aspect of the pericardial sac as medially and as cranially as possible, and subsequently continued cephalad along the right phrenic nerve so that the right lobe of the thymus and surrounding fat are freed up to the point where the left innominate vein joins the superior vena cava. At this stage the origin of the right mammary vein may be dissected free (Figure 4). The 


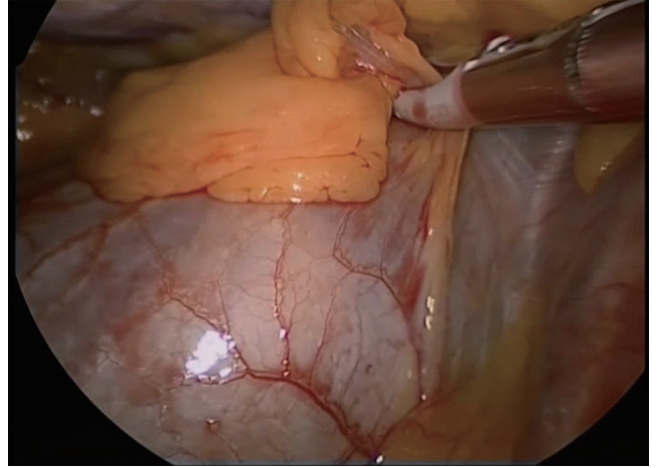

Figure 3 Dissection of the right cardiophrenic fat pad away from right phrenic nerve.

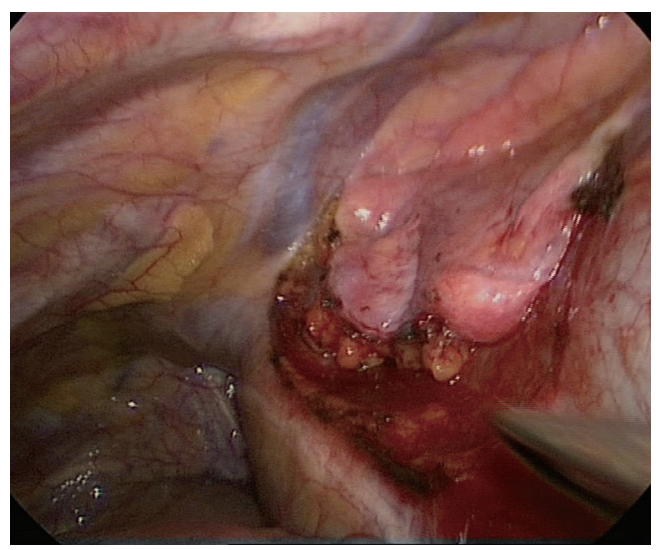

Figure 4 Complete dissection of the right lobe of the thymus. The right mammary vein can be seen joining the right innominate vein at the upper limit of the dissection.

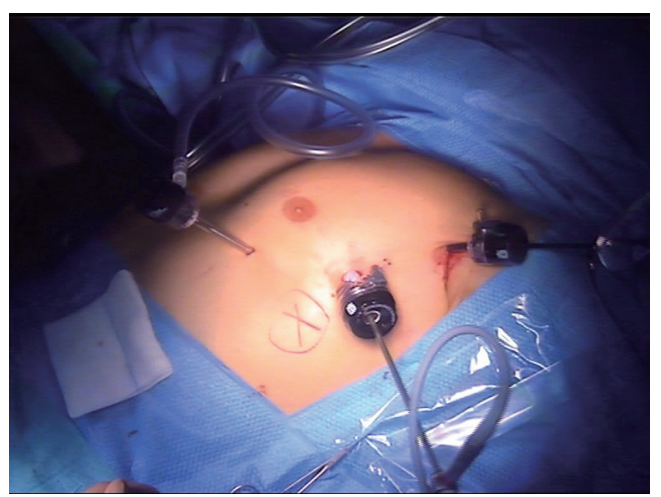

Figure 5 Left-sided approach for non-MG thymoma. Port positioning. MG, myasthenia gravis.

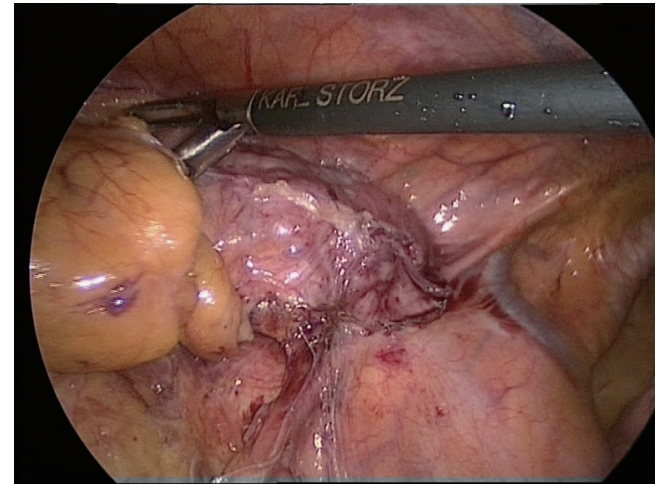

Figure 6 View of the left lobe of the thymus and phrenic nerve. The thymoma was adherent to but not infiltrating the nerve.

single port is closed and the surgeon and assistant move to the left side, while the scrub nurse remains on the right side of the patient. The table will be tiled towards the right side.

\section{Second step: left side}

A $10 \mathrm{~mm}$ trocar is placed in the $5^{\text {th }}$ intercostal space on the mid-axillary line, a second 5 -mm port is placed anteriorly in the inframammary fold, and the last port is placed in the $3^{\text {rd }}$ intercostal space on the mid-axillary line (Figure 5). Occasionally, with larger tumors, the maneuvers may be facilitated by inserting a suction-irrigation tube operated by the assistant through a supplementary 5 -mm port in the $7^{\text {th }}$ or $8^{\text {th }}$ intercostal space on the mid-axillary line. From this moment on, $\mathrm{CO}_{2}$ insufflation will be used throughout the procedure to enlarge the intrapleural space. Insufflation pressure is usually maintained at $8 \mathrm{mmHg}$.

Once again, the dissection starts by grasping and dissecting the fat pad in the cardiophrenic angle anterior to the left phrenic nerve. The fat pad is dissected free from the pericardial sac medially, and from the posterior aspect of the sternum until the previous dissection is reached and the retrosternal space is widely opened.

The dissection is then continued along the left phrenic nerve (Figure 6), by opening the mediastinal pleura up to the thoracic inlet in the proximity of the origin of the mammary vessels. The division of the mediastinal pleura is continued retrosternal towards the right side and then caudally in order to join the previous opening in the retrosternal space.

The upper mediastinum is carefully dissected until the left innominate vein can be seen and freed. Above it, the left cervical horn can be grasped and progressively freed from the innominate vein and from fibrous adhesions in the neck 


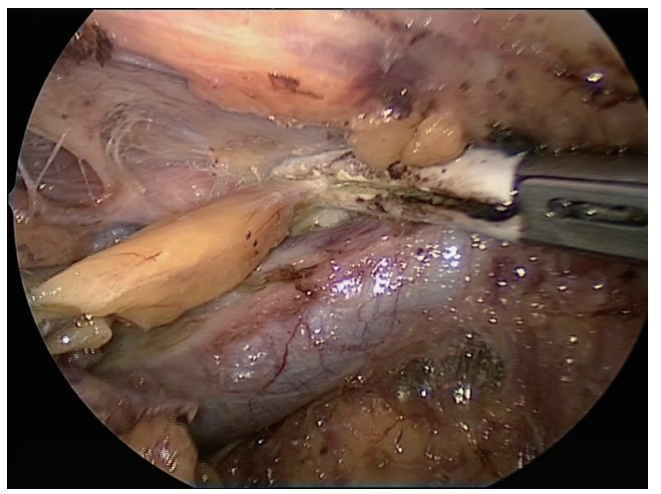

Figure 7 The left cervical horn of the thymus is being dissected in the neck above the left innominate vein and pulled down.

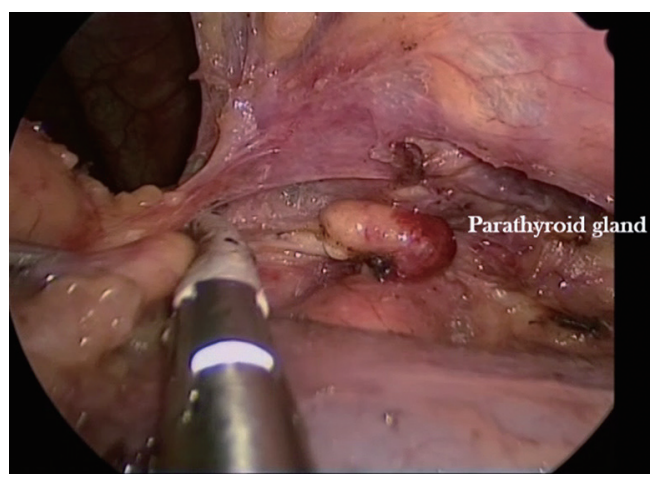

Figure 8 The right cervical horn of the thymus is being dissected in the neck above the left innominate vein (bottom of the picture) and is pulled down in a patient who had previously undergone thyroidectomy. A parathyroid gland is visible after severing of the uppermost fibrous adhesions.

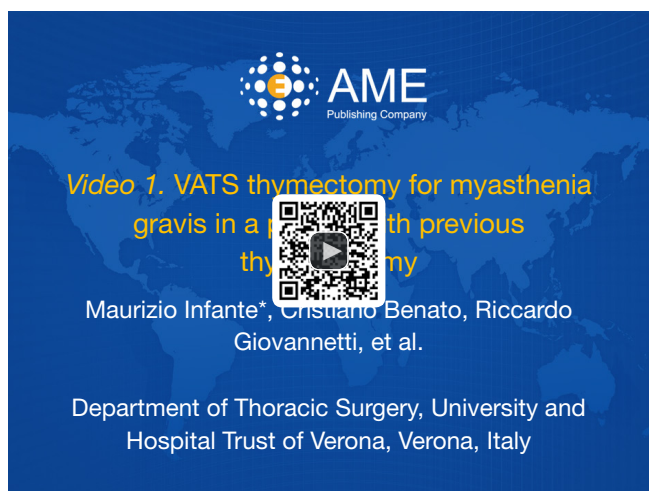

Figure 9 VATS thymectomy for myasthenia gravis in a patient with previous thyroidectomy (25).

Available online: http://www.asvide.com/articles/1754

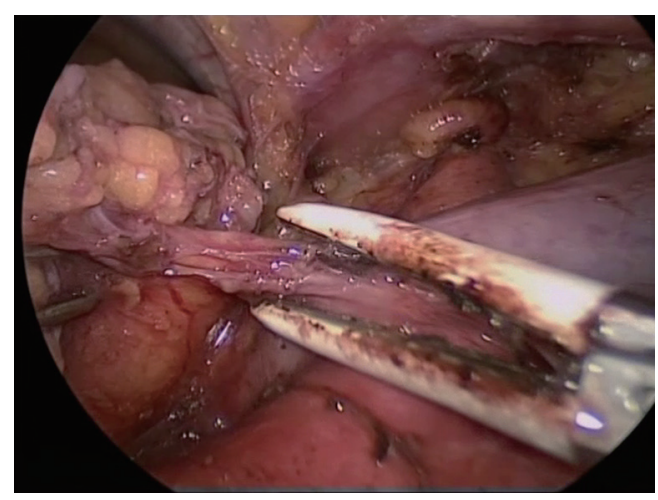

Figure 10 The thymic veins are dissected and can now be interrupted with the radiofrequency grasper.

until it can be mobilized and pulled down into the chest (Figure 7).

The right horn can be now identified and mobilized in a similar manner. There is no risk of recurrent nerve injury in this phase as the dissection is anterior to the trachea (Figure 8), however, in patients who have previously undergone thyroid surgery adhesions in the neck may be found (Figure 9).

It may be advantageous to free the thymus gland from the pericardial sac and the neck alternatively in order to progressively expose the innominate vein along its course from left to right, identify the thymic veins along its lower border, divide them with the energy device (Figure 10) and proceed towards the right side until the right innominate vein is seen joining the superior vena cava. Normally, the previous dissection from the right side will make this final part relatively easy.

The thymus is finally freed from the remaining anterior pericardial adhesions and the whole gland including the right lobe and mediastinal fat is pulled into the left cavity and placed in an endo-bag. It is advantageous to move the camera to the lower port, and enlarge the $10-\mathrm{mm}$ trocar port to extract the specimen.

After a non-complicated VATS thymectomy without associated lung resections, a $16 \mathrm{~F}$ drain is placed in the lowest left-side port to remove fluids that will accumulate in the posterior costophrenic sinus. Normal time for this complete procedure is 2 hours.

\section{Postoperative management}

The patient is extubated in the OR and transferred to an intensive care unit for overnight observation. Under normal circumstances, the patient will be transferred back to the 


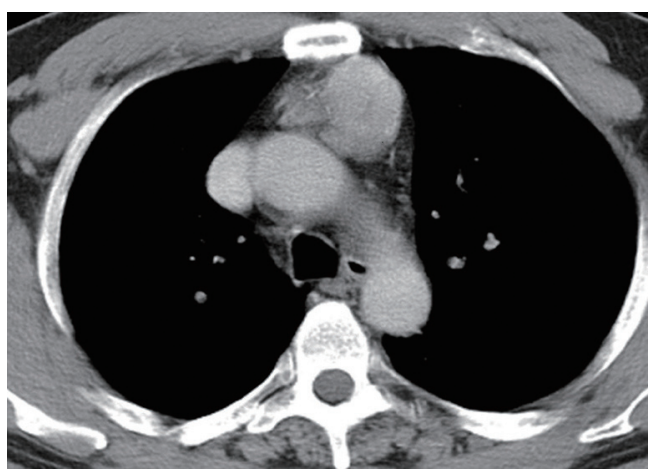

Figure $11 \mathrm{CT}$ scan of the chest showing a $5-\mathrm{cm}$ thymoma with good cleavage plane from the aortic arch that was successfully resected by VATS through the left hemithorax.

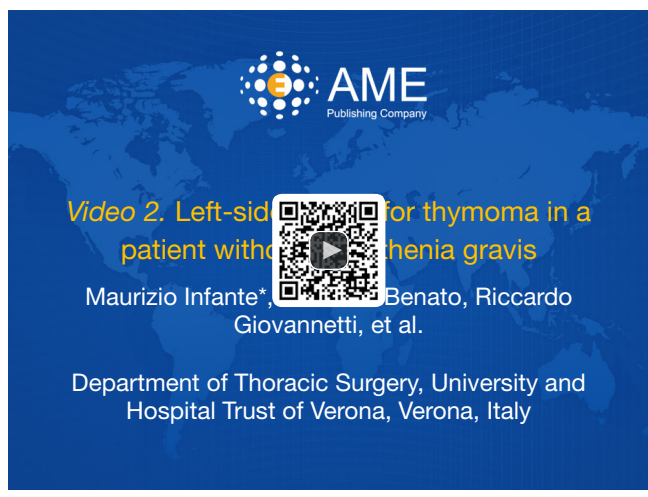

Figure 12 Left-sided VATS for thymoma in a patient without myasthenia gravis (26).

Available online: http://www.asvide.com/articles/1755

regular ward on the subsequent morning. The drain is usually removed and the patient discharged on PO day 2 .

\section{Tips, tricks and pitfalls}

Since stage I-II thymoma may be identified with reasonable accuracy preoperatively, VATS thymectomy is routinely planned in our department in these patients based on CT findings (Figure 11), and conversion to open surgery may be decided after the initial VATS exploration.

A single lumen tube may suffice for patients undergoing VATS-thymectomy for non-thymomatous $\mathrm{MG}$, as $\mathrm{CO}_{2}$ insufflation will keep the lungs away from the anterior mediastinum.

On the other hand, midline thymomas may infiltrate either lung, therefore placing a double lumen tube is advisable when performing VATS thymectomy for thymoma.

Correct positioning of the patient on the operating table and of the trocars is of utmost importance to facilitate surgical maneuvers and minimize operating time in all cases (figures).

A laparoscopic energy device for tissue handling and sealing is instrumental in all phases of the operation. Our preference goes to radiofrequency devices such as the Maryland laparoscopic grasp, as these cause less smoke in the operative field and are less prone to causing unwanted thermal injury in the proximity of the phrenic nerves.

Dissection of the left cardiophrenic fat may be cumbersome-due to the heart bulging towards the camera (Figure 12). Such initial maneuvers may be facilitated by temporarily increasing the intrapleural pressure to $10-12 \mathrm{mmHg}$ for short periods of time if tolerated.

\section{Additional remarks}

Bilateral removal of the whole mediastinal fat in non-MG patients with small thymomas may not be necessary, so that in such patients the right-side phase is skipped altogether, saving time and effort

Although the necessity of removing the entire thymus systematically for small thymomas is debated, MG gravis patients will always require maximal thymectomy, so surgeons should be trained in it. An increased local recurrence rate with thymectomy alone has been recently reported (16,27).

Although VATS thymectomy is a safe procedure in experienced hands, the surgeon must be prepared to convert to an open procedure when there is a substantial risk of vascular injury and be able to handle major bleeding if it occurs during the VATS procedure. Conversion rates in the literature and in our experience are around 6-8\%. In our VATS-thymectomy series all conversions (5/61 patients) were due to oncological limits.

\section{Conclusions}

VATS thymectomy has been refined over time to become a time-sparing, effective and well tolerated procedure, with a low conversion rate, and few complications. It may be appropriate both in non-thymomatous MG and in early stage thymoma as adequate margins and mediastinal fat clearance can be reliably obtained without resorting to the traditional trans-sternal maximal thymectomy-with 
its accompanying burden of potential complications and unsightly scar.

\section{Acknowledgements}

None.

\section{Footnote}

Conflicts of Interest: The authors have no conflicts of interest to declare.

Informed Consent: Written informed consent was obtained from the patients for publication of this manuscript and any accompanying images.

\section{References}

1. Engels EA, Pfeiffer RM. Malignant thymoma in the United States: demographic patterns in incidence and associations with subsequent malignancies. Int J Cancer 2003;105:546-51.

2. Kondo K, Monden Y. Thymoma and myasthenia gravis: a clinical study of 1,089 patients from Japan. Ann Thorac Surg 2005;79:219-24.

3. Ruffini E, Detterbeck F, Van Raemdonck D, et al. Tumours of the thymus: a cohort study of prognostic factors from the European Society of Thoracic Surgeons database. Eur J Cardiothorac Surg 2014;46:361-8.

4. Detterbeck F, Youssef S, Ruffini E, et al. A review of prognostic factors in thymic malignancies. J Thorac Oncol 2011;6:S1698-704.

5. Margaritora S, Cesario A, Cusumano G, et al. Thirty-fiveyear follow-up analysis of clinical and pathologic outcomes of thymoma surgery. Ann Thorac Surg 2010;89:245-52.

6. Fang W, Yao X, Antonicelli A, et al. Comparison of surgical approach and extent of resection for MasaokaKoga Stage I and II thymic tumours in Europe, North America and Asia: an International Thymic Malignancy Interest Group retrospective database analysis. Eur J Cardiothorac Surg 2017. (Epub ahead of print).

7. Jaretzki A 3rd, Wolff M. "Maximal" thymectomy for myasthenia gravis. Surgical anatomy and operative technique. J Thorac Cardiovasc Surg 1988;96:711-6.

8. Sakamaki Y, Oda T, Kanazawa G, et al. Intermediate-term oncologic outcomes after video-assisted thoracoscopic thymectomy for early-stage thymoma. J Thorac Cardiovasc Surg 2014;148:1230-7.e1.
9. Liu TJ, Lin MW, Hsieh MS, et al. Video-assisted thoracoscopic surgical thymectomy to treat early thymoma: a comparison with the conventional transsternal approach. Ann Surg Oncol 2014;21:322-8.

10. Manoly I, Whistance RN, Sreekumar R, et al. Early and mid-term outcomes of trans-sternal and video-assisted thoracoscopic surgery for thymoma. Eur J Cardiothorac Surg 2014;45:e187-93.

11. Ye B, Tantai JC, Ge XX, et al. Surgical techniques for early-stage thymoma: video-assisted thoracoscopic thymectomy versus transsternal thymectomy. J Thorac Cardiovasc Surg 2014;147:1599-603.

12. Zhao Y, Shi J, Fan L, et al. Surgical treatment of thymoma: an 11-year experience with 761 patients. Eur J Cardiothorac Surg 2016;49:1144-9.

13. Yu L, Zhang XJ, Ma S, et al. Thoracoscopic thymectomy for myasthenia gravis with and without thymoma: a singlecenter experience. Ann Thorac Surg 2012;93:240-4.

14. Siwachat S, Tantraworasin A, Lapisatepun $W$, et al. Comparative clinical outcomes after thymectomy for myasthenia gravis: Thoracoscopic versus trans-sternal approach. Asian J Surg 2016. (Epub ahead of print).

15. Nakagawa K, Yokoi K, Nakajima J, et al. Is Thymomectomy Alone Appropriate for Stage I (T1N0M0) Thymoma? Results of a Propensity-Score Analysis. Ann Thorac Surg 2016;101:520-6.

16. Yang Y, Dong J, Huang Y. Thoracoscopic thymectomy Vs. open thymectomy for the treatment of thymoma. A metaanalysis. Eur J Surg Oncol 2016;42:1720-8.

17. Friedant AJ, Handorf EA, Su S, et al. Minimally invasive thymectomy versus open thymectomy fot thymic malignancies: systematic review and meta-analysis. J Thorac Oncol 2016;11:30-8.

18. Shelly S, Agmon-Levin N, Altman A, et al. Thymoma and autoimmunity. Cell Mol Immunol 2011;8:199-202.

19. Nakajima J, Okumura M, Yano M, et al. Myasthenia gravis with thymic epithelial tumour: a retrospective analysis of a Japanese database. Eur J Cardiothorac Surg 2016;49:1510-5.

20. Wolfe GI, Kaminski HJ, Sonnett JR, et al. Randomized Trial of Thymectomy in Myasthenia Gravis. J Thorac Dis 2016;8:E1782-3.

21. Suda T, Kaneda S, Hachimaru A, et al. Thymectomy via a subxiphoid approach: single-port and robot-assisted. J Thorac Dis 2016;8:S265-71.

22. Xie X, Gan X, Chen B, et al. Left- and right-sided videoassisted thoracoscopic thymectomy exhibit similar effects on myasthenia gravis J Thorac Dis 2016;8:124-32.

23. Calhoun RF, Ritter JH, Guthrie TJ, et al. Results of 
Transcervical Thymectomy for Myasthenia Gravis in 100 Consecutive Patients. Ann Surg 1999;230:555-9; discussion 559-61.

24. Hayes SA, Huang J, Plodkowski AJ, et al. Preoperative computed tomography findings predict surgical resectability of thymoma. J Thorac Oncol 2014;9:1023-30.

25. Infante $M$, Benato $C$, Giovannetti $R$, et al. VATS thymectomy for myasthenia gravis in a patient with previous thyroidectomy. Asvide 2017;4:437. Available

doi: 10.21037/jovs.2017.09.01

Cite this article as: Infante $M$, Benato $C$, Giovannetti R, Bonadiman C, Canneto B, Falezza G, Lonardoni A, Gandini P. VATS thymectomy for early stage thymoma and myasthenia gravis: combined right-sided uniportal and left-sided threeportal approach. J Vis Surg 2017;3:144. online: http://www.asvide.com/articles/1754

26. Infante $M$, Benato $C$, Giovannetti R, et al. Left-sided VATS for thymoma in a patient without myasthenia gravis. Asvide 2017;4:438. Available online: http://www.asvide. com/articles/1755

27. Gu Z, Fu J, Shen Y, et al. Thymectomy versus tumor resection for early-stage thymic malignancies: a Chinese Alliance for Research in Thymomas retrospective database analysis. J Thorac Dis 2016;8:680-6. 